\title{
On the Cozero-Divisor Graphs of Commutative Rings
}

\author{
Mojgan Afkhami, Kazem Khashyarmanesh* \\ Department of Pure Mathematics, Ferdowsi University of Mashhad, Mashhad, Iran \\ Email: mojgan.afkhami@yahoo.com, *khashyar@ipm.ir.
}

Received March 22, 2013; revised April 23, 2013; accepted April 30, 2013

Copyright (C) 2013 Mojgan Afkhami, Kazem Khashyarmanesh. This is an open access article distributed under the Creative Commons Attribution License, which permits unrestricted use, distribution, and reproduction in any medium, provided the original work is properly cited.

\section{ABSTRACT}

Let $R$ be a commutative ring with non-zero identity. The cozero-divisor graph of $R$, denoted by $\Gamma^{\prime}(R)$, is a graph with vertices in $W^{*}(R)$, which is the set of all non-zero and non-unit elements of $R$, and two distinct vertices $a$ and $b$ in $W^{*}(R)$ are adjacent if and only if $a \notin b R$ and $b \notin a R$. In this paper, we investigate some combinatorial properties of the cozero-divisor graphs $\Gamma^{\prime}(R[x])$ and $\Gamma^{\prime}(R[[x]])$ such as connectivity, diameter, girth, clique numbers and planarity. We also study the cozero-divisor graphs of the direct products of two arbitrary commutative rings.

Keywords: Clique Number; Connectivity; Cozero-Divisor Graph; Diameter; Direct Product; Girth; Rings of Polynomials; Rings of Power Series.

\section{Introduction}

Let $R$ be a commutative ring with non-zero identity and let $Z(R)$ be the set of zero-divisors of $R$. For an arbitrary subset $A$ of $R$, we put $A^{*}:=A \backslash\{0\}$. The zero-divisor graph of $R$, denoted by $\Gamma(R)$, is an undirected graph whose vertices are elements of $Z^{*}(R)$ with two distinct vertices $a$ and $b$ are adjacent if and only if $a b=0$.

The concept of zero-divisor graph of a commutative ring was introduced by Beck [1], but this work was mostly concerned with colorings of rings. The above definition first appeared in Anderson and Livingston [2], which contained several fundamental results concerning the graph $\Gamma(R)$. The zero-divisor graphs of commutative rings have been studied by several authors. For instance, the preservation and lack thereof of basic properties of $\Gamma(R)$ under extensions to rings of polynomials and power series was studied by Axtell, Coykendall and Stickles in [3] and Lucas in [4]. Also Axtell, Stickles and Warfel in [5], considered the zero-divisor graphs of direct products of commutative rings.

Let $W(R)$ be the set of all non-unit elements of $R$. For an arbitrary commutative ring $R$, the cozero-divisor graph of $R$, denoted by $\Gamma^{\prime}(R)$, was introduced in [6], which is a dual of zero-divisor graph $\Gamma(R)$ "in some sense”. The vertex-set of $\Gamma^{\prime}(R)$ is $W^{*}(R)$ and for

\footnotetext{
"Corresponding author.
}

two distinct vertices $a$ and $b$ in $W^{*}(R), a$ is adjacent to $b$ if and only if $a \notin b R$ and $b \notin a R$, where $c R$ is an ideal generated by the element $c$ in $R$. Some basic results on the structure of this graph and the relations between two graphs $\Gamma(R)$ and $\Gamma^{\prime}(R)$ were studied in [6].

In this paper, we study the cozero-divisor graphs of the rings of polynomials, power series and the direct product of two arbitrary commutative rings. Also, we look at the preservation of the diameter and girth of the cozero-divisor graphs in some extension rings. Our results "in some sense" are the dual of the main results of [3-5].

Throughout the paper, $R$ is a commutative ring with non-zero identity. We denote the set of maximal ideals and the Jacobson radical of $R$ by $\operatorname{Max}(R)$ and $J(R)$, respectively. Also, $U(R)$ is the set of all unit elements of $R$. By a local ring, we mean a (not necessarily Noetherian) ring with a unique maximal ideal.

In a graph $G$, the distance between two distinct vertices $a$ and $b$, denoted by $\mathrm{d}_{G}(a, b)$, is the length of the shortest path connecting $a$ and $b$, if such a path exists; otherwise, we set $\mathrm{d}_{G}(a, b):=\infty$. The diameter of a graph $G$ is

$$
\begin{aligned}
& \operatorname{diam}(G) \\
& =\sup \left\{\mathrm{d}_{G}(a, b) \mid a \text { and } b \text { are distinct vertices of } G\right\} .
\end{aligned}
$$

The girth of $G$, denoted by $g(G)$, is the length of the 
shortest cycle in $G$, if $G$ contains a cycle; otherwise, $g(G):=\infty$. Also, for two distinct vertices $a$ and $b$ in $G$, the notation $a-b$ means that $a$ and $b$ are adjacent. A graph $G$ is said to be connected if there exists a path between any two distinct vertices, and it is complete if it is connected with diameter one. We use $K_{n}$ to denote the complete graph with $n$ vertices. Moreover, we say that $G$ is totally disconnected if no two vertices of $G$ are adjacent. For a graph $G$, let $\chi(G)$ denote the chromatic number of the graph $G$, i.e., the minimal number of colors which can be assigned to the vertices of $G$ in such a way that any two adjacent vertices have different colors. A clique of a graph is any complete subgraph of the graph and the number of vertices in a largest clique of $G$, denoted by clique $(G)$, is called the clique number of $G$. Obviously $\chi(G) \geq$ clique $(G)$ (cf. see [7, p. 289]). For a positive integer $r$, an $r$-partite graph is one whose vertex-set can be partitioned into $r$ subsets so that no edge has both ends in any one subset. A complete $r$-partite graph is one in which each vertex is joined to every vertex that is not in the same subset. The complete bipartite graph (2-partite graph) with subsets containing $m$ and $n$ vertices, respectively, is denoted by $K_{m, n}$. A graph is said to be planar if it can be drawn in the plane so that its edges intersect only at their ends. A subdivision of a graph is any graph that can be obtained from the original graph by replacing edges by paths. A remarkable simple characterization of the planar graphs was given by Kuratowski in 1930. Kuratowski's Theorem says that a graph is planar if and only if it contains no subdivision of $K_{5}$ or $K_{3,3}$ (cf. [8, p. 153]). Also, the valency of a vertex $a$ is the number of edges of the graph $G$ incident with $a$.

\section{Cozero-Divisor Graph of $\boldsymbol{R}[\boldsymbol{x}]$}

In this section, we are going to study some basic properties of the cozero-divisor graph of the polynomial ring $R[x]$. To this end, we first gather together the wellknown properties of the polynomial ring $R[x]$, which are needed in this section.

Remarks 2.1 Let $f(x)=\sum_{i=0}^{n} a_{i} x^{i}$ be an arbitrary element in $R[x]$. Then we have the following statements:

- $f(x)$ is a unit in $R[x]$ if and only if $a_{0}$ is a unit and the coefficients $a_{1}, \cdots, a_{n}$ are nilpotent elements of $R$.

- $f(x)$ is nilpotent if and only if the coefficients $a_{1}, \cdots, a_{n}$ are nilpotent.

- $\mathrm{J}(R[x])=\operatorname{Nil}(R[x])$, where $\operatorname{Nil}(R[x])$ is the nilradical of $R[x]$.

- Since the polynomials $x$ and $1+x$ are non-units, $R[x]$ is a non-local ring.

- By part (i), it is easy to see that $\Gamma^{\prime}(R)$ is an induced subgraph of $\Gamma^{\prime}(R[x])$.
In the following theorem, we show that $\Gamma^{\prime}(R[x])$ is always connected and its diameter is not exceeding three.

Theorem 2.2 The graph $\Gamma^{\prime}(R[x])$ is connected and $\operatorname{diam}\left(\Gamma^{\prime}(R[x])\right) \leq 3$.

Proof. Since $R[x]$ is a non-local ring, by [1, Theorem 2.5], it is enough to show that, for every non-zero element $f(x) \in \mathrm{J}(R[x])$, there exist $m \in \operatorname{Max}(R[x])$ and $g(x) \in m \backslash \mathrm{J}(R[x])$ such that $f(x) \notin g(x) R[x]$. Now, assume that $f(x)$ is a non-zero polynomial in $\mathrm{J}(R[x])$ of degree $t$. Since $x$ is a non-unit element in $R[x]$, there exists a maximal ideal $m$ of $R[x]$ such that $x \in m$. So $x^{t+1} \in m$. On the other hand, by parts (ii) and (iii) of Remarks 2.1, $x^{t+1} \notin \mathrm{J}(R[x])$. Also

$f(x) \notin x^{t+1} R[x]$. Hence the graph $\Gamma^{\prime}(R[x])$ is connected and $\operatorname{diam}\left(\Gamma^{\prime}(R[x])\right) \leq 3$.

The following proposition states that the diameter of $\Gamma^{\prime}(R[x])$ is never one.

Proposition 2.3 The graph $\Gamma^{\prime}(R[x])$ is never complete.

Proof. Clearly $x \in W^{*}(R[x])$ and $\left\{0, x, x^{2}\right\} \subseteq x R[x]$. The claim now follows from [1, Theorem 2.1].

The following corollary is an immediate consequence of Theorem 2.2 and Proposition 2.3.

Corollary 2.4 $\operatorname{diam}\left(\Gamma^{\prime}(R[x])\right)=2$ or 3.

Proposition 2.5 Suppose that $\mathrm{J}(R[x])=0$. Then $\operatorname{diam}\left(\Gamma^{\prime}(R[x])\right)=2$. In particular, if $R$ is reduced, then $\operatorname{diam}\left(\Gamma^{\prime}(R[x])\right)=2$.

Proof. In view of [1, Corollary 2.4],

$\operatorname{diam}\left(\Gamma^{\prime}(R[x])\right) \leq 2$. Now, by Proposition 2.3, one can conclude that $\operatorname{diam}\left(\Gamma^{\prime}(R[x])\right)=2$. Also, if $R$ is reduced, then by Remarks 2.1 (ii), $\operatorname{Nil}(R[x])=0$ and so, by Remarks 2.1 (iii), $\mathrm{J}(R[x])=\operatorname{Nil}(R[x])=0$.

In the next two theorems, we investigate the girth of the graph $\Gamma^{\prime}(R[x])$.

Theorem 2.6 Suppose that $R$ is a non-reduced ring. Then every element of $\mathrm{J}(R[x])$ is in a cycle of length three.

Proof. Assume that $f(x)$ is a non-zero element in $\mathrm{J}(R[x])$ of degree $n$ and consider the elements $x^{t}$ and $1+x^{t}$ in $R[x]$, where $t>n$. Then, by Remarks 2.1 (iv), there exist maximal ideals $m_{1}$ and $m_{2}$ of $R[x]$ such that $x^{t} \in m_{1} \backslash m_{2}$ and $1+x^{t} \in m_{2} \backslash m_{1}$. Since $t>n$, $f(x) \notin x^{t} R[x]$ and $f(x) \notin\left(1+x^{t}\right) R[x]$. Also, by parts (ii) and (iii) of Remarks 2.1, $x^{t}$ and $1+x^{t}$ do not belong to $\mathrm{J}(R[x])$. So, $x^{t} \notin f(x) R[x]$ and $\left(1+x^{t}\right) \notin f(x) R[x]$. Thus, $f(x)$ is adjacent to both distinct vertices $x^{t}$ and $1+x^{t}$. Moreover, it is easy to see that $x^{t}$ is adjacent to $1+x^{t}$. Therefore we have the cycle

$$
f(x)-x^{t}-\left(1+x^{t}\right)-f(x) .
$$

Theorem $2.7 g\left(\Gamma^{\prime}(R[x])\right)=3$.

Proof. Consider the elements $x, 1+x$ and $1+x+x^{2}$ 
in $R[x]$. So there exist two maximal ideals $m_{1}$ and $m_{2}$ in $R[x]$ such that $x \in m_{1} \backslash m_{2}$ and $1+x \in m_{2} \backslash m_{1}$. Hence the vertex $x$ is adjacent to $1+x$. Also, clearly $x \notin\left(1+x+x^{2}\right) R[x]$ and $1+x \notin\left(1+x+x^{2}\right) R[x]$. Now, since the polynomials $x$ and $1+x$ do not divide the polynomial $1+x+x^{2}$, we have that $1+x+x^{2} \notin x R[x]$ and $1+x+x^{2} \notin(1+x) R[x]$. Hence

$$
\left(1+x+x^{2}\right)-(1+x)-x-\left(1+x+x^{2}\right)
$$

is the required cycle.

In the next theorem we study the clique number of $\Gamma^{\prime}(R[x])$.

Theorem 2.8 In the graph $\Gamma^{\prime}(R[x])$, clique $\left(\Gamma^{\prime}(R[x])\right)$ is infinity and hence the chromatic number $\chi\left(\Gamma^{\prime}(R[x])\right)$ is infinity.

Proof. Let $n$ be a positive integer and consider the subgraph $G_{n}$ of $\Gamma^{\prime}(R[x])$ with vertex-set

$\left\{1+x+\cdots+x^{t} \mid=n, \cdots, 2 n\right\}$. Now, for every two distinct polynomials $1+x+\cdots+x^{i}$ and $1+x+\cdots+x^{j}$ with $i<$ $j$, clearly we have that

$$
1+x+\cdots+x^{i} \notin\left(1+x+\cdots+x^{j}\right) R[x] .
$$

Also, since $n \leq i, j \leq 2 n$, we have that $j-2 i<1$. This means that $j-i-1<i$, and so $1+x+\cdots+x^{i}$ does not divide the polynomial $1+x+\cdots+x^{j}$. Thus

$1+x+\cdots+x^{j} \notin\left(1+x+\cdots+x^{i}\right) R[x]$. Hence, $G_{n}$ is a complete subgraph of $\Gamma^{\prime}(R[x])$ which is isomorphic to $K_{n+1}$. So clique $\left(\Gamma^{\prime}(R[x])\right)$ is infinity. This implies that $\chi\left(\Gamma^{\prime}(R[x])\right)$ is infinity.

Theorem 2.9 The cozero-divisor graph $\Gamma^{\prime}(R[x])$ is not planar.

Proof. In view of the proof of Theorem 2.8, for all positive integers $n$, the cozero-divisor graph $\Gamma^{\prime}(R[x])$ has a complete subgraph isomorphic to $K_{n}$. In particular, for $n=5$, the graph $K_{5}$ is a subgraph of $\Gamma^{\prime}(R[x])$. So, by Kuratowski's Theorem (cf. [8, p. 153]), $\Gamma^{\prime}(R[x])$ is not planar.

Recall that a graph on $n$ vertices such that $n-1$ of the vertices have valency one, all of which are adjacent only to the remaining vertex $a$, is called a star graph with center $a$. Also, a refinement of a graph $H$ is a graph $G$ such that the vertex-sets of $G$ and $H$ are the same and every edge in $H$ is an edge in $G$. Now, we have the following result.

Proposition 2.10 If there exists a maximal ideal $m$ of $R$ with $|m|=2$, then there is a refinement of a star graph in the structure of $\Gamma^{\prime}(R[x])$.

Proof. Suppose that $m=\{0, a\}$ is a maximal ideal of $R$. Then, for every element $b \in W^{*}(R)$ with $a \neq b$, we have that $b \notin a R$. Also $a \notin b R$. Hence, $a$ is adjacent to $b$. Therefore, $\Gamma^{\prime}(R)$ is a refinement of a star graph with center $a$. Now, by Remarks $2.1(\mathrm{v}), \Gamma^{\prime}(R)$ is an in- duced subgraph of $\Gamma^{\prime}(R[x])$. So $\Gamma^{\prime}(R[x])$ contains a refinement of a star graph.

\section{Cozero-Divisor Graph of $\boldsymbol{R}[[\boldsymbol{x}]]$}

We begin this section with some elementary remarks about the rings of power series which may be valuable in turn. These facts can be immediately gained from the elementary notes about power series.

\section{Remarks 3.1}

- $f(x)=\sum_{i=0}^{\infty} a_{i} x^{i}$ is a unit in $R[[x]]$ if and only if $a_{0}$ is a unit in $R$.

- $f(x)=\sum_{i=0}^{\infty} a_{i} x^{i}$ belongs to the Jacobson radical of $R[[x]]$ if and only if $a_{0}$ belongs to the Jacobson radical of $R$.

- $R[[x]]$ is a local ring if and only if $R$ is local.

- The cozero-divisor graph $\Gamma^{\prime}(R)$ is an induced subgraph of $\Gamma^{\prime}(R[[x]])$, but $\Gamma^{\prime}(R[x])$ is not a subgraph of $\Gamma^{\prime}(R[[x]])$, since $1+x$ is a vertex of $\Gamma^{\prime}(R[x])$ but it is not in the vertex-set of $\Gamma^{\prime}(R[[x]])$.

In the following proposition, we study the connectivity and diameter of $\Gamma^{\prime}(R[[x]])$, whenever $R$ is non-local.

Proposition 3.2 Let $R$ be a non-local ring. Then the cozero-divisor graph $\Gamma^{\prime}(R[[x]])$ is connected and $\operatorname{diam}\left(\Gamma^{\prime}(R[[x]])\right) \leq 3$.

Proof. Suppose that $f(x)=\sum_{i=0}^{\infty} a_{i} x^{i}$ is a non-zero element in $J(R[[x]])$. By [6, Theorem 2.5], it is enough to show that $f(x)$ is adjacent to some element in $W(R[[x]]) \backslash J(R[[x]])$. In this regard, we have the following two cases:

Case 1. Assume that $a_{i} \in U(R)$, for some $i \geq 1$ and consider an element $b$ in $W(R) \backslash J(R)$. We will show that $f(x)$ is adjacent to $b$. Clearly, by Remarks 3.1 (i), (ii), $b \in W(R[[x]]) \backslash J(R[[x]])$. Now, assume in contrary that $b \in f(x) R[[x]]$ and look for a contradiction. We have that $b=f(x) g(x)$, for some $g(x)=\Sigma_{i=0}^{\infty} g_{i} x^{i}$ in $R[[x]]$. Since, by Remarks 3.1 (ii), $a_{0} \in J(R)$, we have that $b \in J(R)$ which is impossible. Also, if $f(x) \in$ $b R[[x]]$, then $a_{i}=b g_{i}$, for some non-zero element $g_{i}$ in $R$, which is impossible. Therefore $f(x)$ and $b$ are adjacent.

Case 2. Suppose that $a_{i} \in W(R)$, for all $i \geq 1$. First assume that $a_{i} \notin J(R)$, for some $i \geq 1$. Hence, there exist maximal ideals $m$ and $m^{\prime}$ such that $a_{i} \in m \backslash m^{\prime}$. By considering an element $b$ in $m^{\prime} \backslash m$, one can conclude that $a_{i}$ is adjacent to $b$. Now if $f(x) \in b R[[x]]$, then $a_{i}=b g_{i}$, for some non-zero element $g_{i}$ in $R$ which is a contradiction because the vertices $a_{i}$ and $b$ are adjacent. On the other hand, $b \notin f(x) R[[x]]$. Thus the vertices $f(x)$ and $b$ are adjacent.

Now, let $a_{i} \in J(R)$, for all $i \geq 1$. Choose $b \in W(R) \backslash J(R)$. Hence $b \in W(R[[x]]) \backslash J(R[[x]])$. 
We claim that $f(x)$ is adjacent to $b+x^{t+1}$, where $t$ is the least non-zero power of $x$ in the polynomial $f(x)$. Clearly, $f(x) \notin\left(b+x^{t+1}\right) R[[x]]$. Now, if $b+x^{t+1}=$ $f(x) g(x)$ for some $g(x)=\Sigma_{i=0}^{\infty} g_{i} x^{i}$ in $R[[x]]$, then

$$
1=a_{0} g_{t+1}+\cdots+a_{t+1} g_{0},
$$

which belongs to $J(R)$ and this is impossible. Hence, we have that $f(x)$ is adjacent to $b+x^{t+1}$.

Therefore $\Gamma^{\prime}(R[[x]])$ is connected and also, by considering the above cases, it is routine to check that $\operatorname{diam}\left(\Gamma^{\prime}(R[[x]])\right) \leq 3$.

In the next lemma, we investigate the adjacency in $\Gamma^{\prime}(R[[x]])$ in the case that $R$ is a local ring.

Lemma 3.3 Assume that $R$ is a local ring with maximal ideal m. Let $f(x)=\sum_{i=0}^{\infty} a_{i} x^{i}$ be a non-zero element in $J(R[[x]])$. Then we have the following statements:

- if $a_{0} \neq 0$, then $f(x)$ is adjacent to $x$;

- if $a_{0}=0$ and $a_{i} \in U(R)$, for some $i \geq 1$, then $f(x)$ is adjacent to all non-zero elements of $J(R)$; and,

- if $a_{0}=0$ and $a_{i} \in \mathrm{m}$, for all $i \geq 1$, then $f(x)$ is adjacent to $x^{t+1}$, where $t$ is the least non-zero power of $x$ in $f(x)$.

Proof. 1) Assume on the contrary that $x=f(x) g(x)$, where $g(x)=\sum_{i=0}^{\infty} g_{i} x^{i}$. Then $a_{0} g_{0}=0$ and

$1=a_{0} g_{1}+g_{0} a_{1}$. Since $a_{0}$ and $g_{0}$ belong to $m$, we have that $1 \in \mathrm{m}$ which is a contradiction. Also we have that $f(x) \notin x R[[x]]$. Thus $f(x)$ is adjacent to $x$.

2) Let $b$ be a non-zero element in $m$. Then if $f(x) \in b R[[x]]$, we conclude that $a_{i} \in m$ which is impossible. So $f(x) \notin b R[[x]]$. Now, since $a_{0}=0$, $b \notin f(x) R[[x]]$. Therefore, $f(x)$ is adjacent to $b$.

3) Clearly, since $t$ is the least non-zero power of $x$ in $f(x), f(x) \notin x^{t+1} R[[x]]$. Moreover, if $x^{t+1}=f(x) g(x)$, for some $g(x)=\sum_{i=0}^{\infty} g_{i} x^{i}$ in $R[[x]]$, then

$$
1=a_{0} g_{t+1}+\cdots+a_{t+1} g_{0} .
$$

This means that $1 \in \mathrm{m}$ which is impossible. Hence $x^{t+1} \notin f(x) R[[x]]$. So $f(x)$ is adjacent to $x^{t+1}$.

The following result, which is one of our main results in this section, states that $\Gamma^{\prime}(R[[x]])$ is connected and the diameter of $\Gamma^{\prime}(R[[x]])$ is not exceeding four.

Theorem 3.4 The cozero-divisor graph $\Gamma^{\prime}(R[[x]])$ is always connected and also $\operatorname{diam}\left(\Gamma^{\prime}(R[[x]])\right) \leq 4$.

Proof. Owing to Proposition 3.2, the result holds in the case that $R$ is non-local. Assume that $R$ is a local ring with maximal ideal $m$. In view of part (iii) of Remarks 3.1, $R[[x]]$ is also a local ring. Now, let

$f(x)=\sum_{i=0}^{\infty} a_{i} x^{i}$ and $g(x)=\Sigma_{i=0}^{\infty} b_{i} x^{i}$ be two non-zero elements in $W(R[[x]])$ that are not adjacent. We have the following cases for consideration:

Case 1. $a_{0} \neq 0$ and $b_{0} \neq 0$. Then by Lemma 3.3 (i), we have that $f(x)-x-g(x)$.

Case 2. $a_{0}=0=b_{0}$. If $a_{i}, b_{j} \in U(R)$, for some $i, j$, then by part (ii) of Lemma 3.3, $f(x)-c-g(x)$, for all non-zero elements $c$ in $m$.

Also, if $a_{i}, b_{j} \in m$, for all $i, j$, and $t, t^{\prime}$ are the least non-zero powers of $x$ in $f(x)$ and $g(x)$, respectively, with $t^{\prime} \leq t$, then by Lemma 3.3 (iii), one can easily check that $f(x)-x^{t+1}-g(x)$.

Finally, we may assume that for some positive integer $i, a_{i} \in U(R)$ and $b_{j} \in m$, for all $j$. Thus, by parts (ii) and (iii) of Lemma 3.3, we have the path

$f(x)-c-x^{t+1}-g(x)$, where $c$ is a non-zero element in $m$ and $t$ is the least non-zero power of $x$ in $g(x)$.

Case 3. Without loss of generality, we may assume that $a_{0} \neq 0$ and $b_{0}=0$. So, if $b_{j} \in U(R)$, for some $j$, then in view of parts (i) and (ii) of Lemma 3.3, we have the path $f(x)-x-c-g(x)$, where $c$ is a non-zero element in $m$.

Moreover, if $b_{j} \in m$, for all $j$, then by Lemma 3.3, we have $f(x)-x-c-x^{t+1}-g(x)$, where $c$ is a non-zero element in $m$ and $t$ is the least non-zero power of $x$ in $g(x)$.

Therefore, the cozero-divisor graph $\Gamma^{\prime}(R[[x]])$ is connected and in view of the above cases, one can easily check that $\operatorname{diam}\left(\Gamma^{\prime}(R[[x]])\right) \leq 4$.

The following lemma is needed in the sequel.

Lemma 3.5 Let $a \in W^{*}(R)$ and let $i$ and $j$ be positive integers such that $i<j<2 i$. Then the vertices $a+x^{i}$ and $a+x^{j}$ are adjacent in $\Gamma^{\prime}(R[[x]])$.

Proof. Suppose to the contrary that $a+x^{j}=\left(a+x^{i}\right) f(x)$, where

$f(x)=b_{0}+b_{1} x+\cdots+b_{j-i} x^{j-i}$ is a non-zero polynomial in $R[[x]]$. So, we have $a b_{0}=a$ and $b_{0}=0$. Thus $a=0$ which is a contradiction. Hence $a+x^{j} \notin\left(a+x^{i}\right) R[[x]]$. Also, clearly $a+x^{i} \notin\left(a+x^{j}\right) R[[x]]$. So the vertices $a+x^{i}$ and $a+x^{j}$ are adjacent in the cozero-divisor graph $\Gamma^{\prime}(R[[x]])$.

In the next theorem, we show that $\mathrm{g}\left(\Gamma^{\prime}(R[[x]])\right)=3$.

Theorem 3.6 The cozero-divisor graph $\Gamma^{\prime}(R[[x]])$ has girth three.

Proof. Let $a \in W^{*}(R)$. Consider the elements $x$, $a+x^{2}$ and $a+x^{3}$ in $R[[x]]$. Clearly, $x \notin\left(a+x^{2}\right) R[[x]]$ and $x \notin\left(a+x^{3}\right) R[[x]]$. Also, since $a \neq 0, a+x^{2}$ and $a+x^{3}$ don't belong to $x R[[x]]$. Hence, we have the following path

$$
\left(a+x^{2}\right)-x-\left(a+x^{3}\right) .
$$

Now, in view of Lemma 3.5, one can conclude that $a+x^{2}$ and $a+x^{3}$ are adjacent. Therefore, we have the cycle $x-\left(a+x^{2}\right)-\left(a+x^{3}\right)-x$. Hence, $\mathrm{g}\left(\Gamma^{\prime}(R[[x]])\right)=3$.

In the next theorem, we compute the clique number of $\Gamma^{\prime}(R[[x]])$.

Theorem 3.7 In the graph $\Gamma^{\prime}(R[[x]])$, 
clique $\left(\Gamma^{\prime}(R[[x]])\right)$ is infinity and hence $\chi\left(\Gamma^{\prime}(R[[x]])\right)$ is also infinity.

Proof. For every positive integer $n$, it is enough to construct a complete subgraph of $\Gamma^{\prime}(R[[x]])$ with $n$ vertices. To this end, let $n$ be an arbitrary positive integer and $a \in W^{*}(R)$. Then, by Lemma 3.5, it is easy to see that the subgraph with vertex-set

$\left\{a+x^{n+1}, \cdots, a+x^{2 n}\right\}$ is a complete subgraph of

$\Gamma^{\prime}(R[[x]])$ which is isomorphic to $K_{n}$. So

clique $\left(\Gamma^{\prime}(R[[x]])\right)$ is infinity and this implies that $\chi\left(\Gamma^{\prime}(R[[x]])\right)$ is infinity.

We end this section with the following theorem.

Theorem 3.8 The cozero-divisor graph $\Gamma^{\prime}(R[[x]])$ is not planar.

Proof. In view of the proof of Theorem 3.7, $K_{5}$ is a subgraph of $\Gamma^{\prime}(R[[x]])$. Thus, by Kuratowski's Theorem, $\Gamma^{\prime}(R[[x]])$ is not planar.

\section{Cozero-Divisor Graph of $R_{1} \times R_{2}$}

Throughout this section, $R_{1}$ and $R_{2}$ are two commutative rings with non-zero identities. We will study the cozerodivisor graph of the direct product of $R_{1}$ and $R_{2}$. Note that an element $(a, b)$ belongs to $W\left(R_{1} \times R_{2}\right)$ if and only if $a \in W\left(R_{1}\right)$ or $b \in W\left(R_{2}\right)$. We begin this section with the following lemma.

Lemma 4.1 Suppose that $R=R_{1} \times \cdots \times R_{n}$ is a direct product of finite commutative rings. If $a_{i}$ is adjacent to $b_{i}$ in $\Gamma^{\prime}\left(R_{i}\right)$, for some $1 \leq i \leq n$, then every element in $R$ with $i$-th component $a_{i}$ is adjacent to all elements in $R$ with $i$-th component $b_{i}$.

Proof. Suppose that $a_{i}$ is adjacent to $b_{i}$ in $\Gamma^{\prime}\left(R_{i}\right)$ and assume on the contrary that the vertices $\left(a_{1}, \cdots, a_{n}\right)$ and $\left(b_{1}, \cdots, b_{n}\right)$ are not adjacent in $\Gamma^{\prime}(R)$. Without loss of generality, suppose that

$\left(a_{1}, \cdots, a_{n}\right) \in\left(b_{1}, \cdots, b_{n}\right)\left(R_{1} \times \cdots \times R_{n}\right)$. Thus

$\left(a_{1}, \cdots, a_{n}\right)=\left(b_{1}, \cdots, b_{n}\right)\left(r_{1}, \cdots, r_{n}\right)$, for some non-zero element $\left(r_{1}, \cdots, r_{n}\right) \in R$. Therefore $a_{i}=r_{i} b_{i}$ and hence $a_{i}$ is not adjacent to $b_{i}$, which is a contradiction.

The following corollary follows immediately from Lemma 4.1.

Corollary 4.2 Suppose that $(a, b)$, $\left(a^{\prime}, b^{\prime}\right) \in W^{*}\left(R_{1}\right) \times W^{*}\left(R_{2}\right)$ such that they are not adjacent in $\Gamma^{\prime}\left(R_{1} \times R_{2}\right)$. Then $a$ is not adjacent to $a^{\prime}$ in $\Gamma^{\prime}\left(R_{1}\right)$ and $b$ is not adjacent to $b^{\prime}$ in $\Gamma^{\prime}\left(R_{2}\right)$.

In the next lemma, we establish some relations between the adjacency in the graph $\Gamma^{\prime}\left(R_{1} \times R_{2}\right)$ and adjacency in both graphs $\Gamma^{\prime}\left(R_{1}\right)$ and $\Gamma^{\prime}\left(R_{2}\right)$.

\section{Lemma 4.3}

- Let $a \in R_{1}$ and $b, b^{\prime} \in R_{2}$. Then $(a, b)$ is adjacent to $\left(a, b^{\prime}\right)$ in $\Gamma^{\prime}\left(R_{1} \times R_{2}\right)$ if and only if $b$ is adjacent to $b^{\prime}$ in $\Gamma^{\prime}\left(R_{2}\right)$.
- Let $b \in R_{2}$ and $a, a^{\prime} \in R_{1}$. Then $(a, b)$ is adjacent to $\left(a^{\prime}, b\right)$ in $\Gamma^{\prime}\left(R_{1} \times R_{2}\right)$ if and only if $a$ is adjacent to $a^{\prime}$ in $\Gamma^{\prime}\left(R_{1}\right)$.

Proof. 1) Suppose that $(a, b)$ is adjacent to $\left(a, b^{\prime}\right)$. Note that if at least one of the elements $b$ or $b^{\prime}$ is zero or unit, then $(a, b)$ is not adjacent to $\left(a, b^{\prime}\right)$ in $\Gamma^{\prime}\left(R_{1} \times R_{2}\right)$. Thus we can suppose that $b, b^{\prime} \in W^{*}\left(R_{2}\right)$. Now, if $b$ is not adjacent to $b^{\prime}$, then $b \in b^{\prime} R_{2}$ or $b^{\prime} \in b R_{2}$. So without loss of generality, we may assume that $b=r b^{\prime}$ for some non-zero element $r \in R_{2}$. Hence $(a, b)=(1, r)\left(a, b^{\prime}\right)$. This means that $(a, b)$ and $\left(a, b^{\prime}\right)$ are not adjacent in $\Gamma^{\prime}\left(R_{1} \times R_{2}\right)$ which is impossible. Therefore $b$ and $b^{\prime}$ are adjacent in $\Gamma^{\prime}\left(R_{2}\right)$. Conversely, if $b$ is adjacent to $b^{\prime}$, then by Lemma 4.1, we have that $(a, b)$ is adjacent to $\left(a, b^{\prime}\right)$.

2) The proof is similar to part 1 ).

The following propositions follow directly from Lemma 4.3.

Proposition 4.4 Assume that either $\Gamma^{\prime}\left(R_{1}\right)$ or $\Gamma^{\prime}\left(R_{2}\right)$ is not planar. Then $\Gamma^{\prime}\left(R_{1} \times R_{2}\right)$ is not planar.

Proof. Without loss of generality, suppose that $\Gamma^{\prime}\left(R_{1}\right)$ is not planar. So, by Kuratowski's Theorem (cf. [8, p. 153]), it contains a subdivision of $K_{5}$ or $K_{3,3}$. Now, by Lemma 4.3 (ii), one can conclude that $\Gamma^{\prime}\left(R_{1} \times R_{2}\right)$ is not planar.

Proposition 4.5 In $\Gamma^{\prime}\left(R_{1} \times R_{2}\right)$, we have the following inequalities:

- $\operatorname{clique}\left(\Gamma^{\prime}\left(R_{1} \times R_{2}\right)\right)$

$$
\geq \operatorname{Max}\left\{\operatorname{clique}\left(\Gamma^{\prime}\left(R_{1}\right)\right) \text {, clique }\left(\Gamma^{\prime}\left(R_{2}\right)\right)\right\} \text {; }
$$

- $\chi\left(\Gamma^{\prime}\left(R_{1} \times R_{2}\right)\right) \geq \operatorname{Max}\left\{\chi\left(\Gamma^{\prime}\left(R_{1}\right)\right), \chi\left(\Gamma^{\prime}\left(R_{2}\right)\right)\right\}$.

Remark 4.6 Suppose that $a \in R_{1}^{*}$ and $b \in R_{2}^{*}$. Then $(a, 0)$ is adjacent to $(0, b)$.

In the following theorem, we invoke the previous lemmas to show that $\Gamma^{\prime}\left(R_{1} \times R_{2}\right)$ is a complete bipartite graph whenever $R_{1}$ and $R_{2}$ are fields.

Theorem 4.7 Assume that $R_{1}$ and $R_{2}$ are fields. Then $\Gamma^{\prime}\left(R_{1} \times R_{2}\right)$ is a complete bipartite graph.

Proof. Put $V_{1}:=\left\{(a, 0) \mid a \in R_{1}^{*}\right\}$ and $V_{2}:=\left\{(0, b) \mid b \in R_{2}^{*}\right\}$. Clearly $V_{1} \cup V_{2}=W^{*}\left(R_{1} \times R_{2}\right)$. By

Remark 4.6, every element in $V_{1}$ is adjacent to all elements of $V_{2}$ and vice versa. Also, it is easy to see that there is no adjacency between vertices in $V_{1}$ (or $V_{2}$ ). So $\Gamma^{\prime}\left(R_{1} \times R_{2}\right)$ is a complete bipartite graph.

Corollary 4.8 Let $\mathbb{F}$ be an arbitrary field. Then $\Gamma^{\prime}\left(\mathbb{Z}_{2} \times \mathbb{F}\right)$ and $\Gamma^{\prime}\left(\mathbb{F} \times \mathbb{Z}_{2}\right)$ are star graphs.

Remark 4.9 It is easy to see that $(a, b)$ is adjacent to $\left(0, b^{\prime}\right)$ in $\Gamma^{\prime}\left(R_{1} \times R_{2}\right)$, for any $a \in R_{1}^{*}, b \in W\left(R_{2}\right)$ and $b^{\prime} \in U\left(R_{2}\right)$. Similarly, $(a, b)$ is adjacent to $\left(a^{\prime}, 0\right)$ $\Gamma^{\prime}\left(R_{1} \times R_{2}\right)$, for any $a \in W\left(R_{1}\right), \quad b \in R_{2}^{*}$ and $a^{\prime} \in U\left(R_{1}\right)$.

The following theorem is one of our main results in this section. 
Theorem 4.10 The cozero-divisor graph $\Gamma^{\prime}\left(R_{1} \times R_{2}\right)$ is connected and $\operatorname{diam}\left(\Gamma^{\prime}\left(R_{1} \times R_{2}\right)\right) \leq 3$.

Proof. Suppose that $(a, b)$ and $\left(a^{\prime}, b^{\prime}\right)$ are arbitrary elements in $W^{*}\left(R_{1} \times R_{2}\right)$. We have the following cases for consideration:

Case 1. $a, a^{\prime} \in W\left(R_{1}\right)$. If $b=0=b^{\prime}$, then consider the path $(a, 0)-(0,1)-\left(a^{\prime}, 0\right)$. If $b \neq 0$ and $b^{\prime} \neq 0$, then, by Remark 4.9, we have that $(a, b)-(1,0)-\left(a^{\prime}, b^{\prime}\right)$. Now, suppose that $b \neq 0$ and $b^{\prime}=0$. Then, in view of Remarks 4.6 and 4.9, one can obtain the path $(a, b)-(1,0)-(0,1)-\left(a^{\prime}, 0\right)$ in $\Gamma^{\prime}\left(R_{1} \times R_{2}\right)$. The similar result holds in the case that $b^{\prime} \neq 0$ and $b=0$.

Case 2. $a \in W\left(R_{1}\right)$ and $a^{\prime} \notin W\left(R_{1}\right)$. If $b \neq 0$, then, by Remark 4.9 , whenever $b^{\prime}=0$, we have that $(a, b)$ $\left(a^{\prime}, b^{\prime}\right)$. Otherwise, $b^{\prime} \neq 0$. Since $\left(a^{\prime}, b^{\prime}\right) \in W^{*}\left(R_{1} \times R_{2}\right)$ and $a^{\prime} \notin W\left(R_{1}\right)$, we have $b^{\prime} \in W\left(R_{2}\right)$. Now, if $(0,1) \in$ $\left(a^{\prime}, b^{\prime}\right)\left(R_{1} \times R_{2}\right)$, then $1 \in b^{\prime} R_{2}$. But $b^{\prime} R_{2} \subseteq W\left(R_{2}\right)$ and this implies that $1 \in W\left(R_{2}\right)$ which is not true. Hence $(0,1) \notin\left(a^{\prime}, b^{\prime}\right)\left(R_{1} \times R_{2}\right)$. Also, since $a^{\prime} \neq 0$, it is easy to see that $\left(a^{\prime}, b^{\prime}\right) \notin(0,1)\left(R_{1} \times R_{2}\right)$. Therefore, we have the path $(a, b)-(1,0)-(0,1)-\left(a^{\prime}, b^{\prime}\right)$. Also, if $b=0$, then by Remarks 4.6 and 4.9 , we can consider the path $(a, 0)-(0,1)-\left(a^{\prime}, b^{\prime}\right)$. The similar result holds if $a \notin$ $W\left(R_{1}\right)$ and $a^{\prime} \in W\left(R_{1}\right)$.

Case 3. $a, a^{\prime} \notin W\left(R_{1}\right)$. Then we have that

$b, b^{\prime} \in W\left(R_{2}\right)$, and we can apply Case 1 on the second component of ordered pairs.

Now, in view of the above cases, it is easy to see that $\operatorname{diam}\left(\Gamma^{\prime}\left(R_{1} \times R_{2}\right)\right) \leq 3$.

In the next proposition, we provide a characterization of the complete cozero-divisor graph $\Gamma^{\prime}\left(R_{1} \times R_{2}\right)$.

Proposition 4.11 The graph $\Gamma^{\prime}\left(R_{1} \times R_{2}\right)$ is complete if and only if $R_{1} \times R_{2}$ is isomorphic to $\mathbb{Z}_{2} \times \mathbb{Z}_{2}$.

Proof. If $\left|R_{1}\right|>2$, then $(1,0)$ is not adjacent to $(a, 0)$, for some $1 \neq a \in R_{1}^{*}$. Similarly, if $\left|R_{2}\right|>2$, then $\Gamma^{\prime}\left(R_{1} \times R_{2}\right)$ is not complete. So, if $\Gamma^{\prime}\left(R_{1} \times R_{2}\right)$ is complete, then $R_{1} \times R_{2} \cong \mathbb{Z}_{2} \times \mathbb{Z}_{2}$. Also, clearly the graph $\Gamma^{\prime}\left(\mathbb{Z}_{2} \times \mathbb{Z}_{2}\right)$ is complete.

Corollary 4.12 If $R_{1} \times R_{2} \neq \mathbb{Z}_{2} \times \mathbb{Z}_{2}$, then $\operatorname{diam}\left(\Gamma^{\prime}\left(R_{1} \times R_{2}\right)\right)=2$ or 3 .

In the following theorem, we study the girth of

$\Gamma^{\prime}\left(R_{1} \times R_{2}\right)$. Note that we consider $\Gamma^{\prime}\left(\mathbb{Z}_{2}\right)$ to be totally disconnected.

\section{Theorem 4.13}

- If at least one of the cozero-divisor graph $\Gamma^{\prime}\left(R_{1}\right)$ or $\Gamma^{\prime}\left(R_{2}\right)$ is not totally disconnected, then $\mathrm{g}\left(\Gamma^{\prime}\left(R_{1} \times R_{2}\right)\right)=3$.

- If $R_{1} \neq \mathbb{Z}_{2}$ and $R_{2} \neq \mathbb{Z}_{2}$, then $\mathrm{g}\left(\Gamma^{\prime}\left(R_{1} \times R_{2}\right)\right) \leq 4$.

- If $R_{1}=\mathbb{Z}_{2}$ and $R_{2}$ is a field, then $g\left(\Gamma^{\prime}\left(R_{1} \times R_{2}\right)\right)=\infty$.

- If $R_{1}=\mathbb{Z}_{2}$ and $R_{2}$ is not a field, then $g\left(\Gamma^{\prime}\left(R_{1} \times R_{2}\right)\right)=3,4$ or $\infty$.

Proof. 1) Without loss of generality, suppose that $a, b \in W^{*}\left(R_{2}\right)$ such that $a$ is adjacent to $b$. Now, by Lemma 4.3 (i) and Remark 4.9, we have the cycle
$(0,1)-(1, a)-(1, b)-(0,1)$ in $\Gamma^{\prime}\left(R_{1} \times R_{2}\right)$.

2) Let $a \in R_{1}^{*}$ and $b \in R_{2}^{*}$ such that $a$ and $b$ are not identity. Now, consider the cycle $(a, 0)-(0,1)-(1,0)-(0, b)-(a, 0)$ in $\Gamma^{\prime}\left(R_{1} \times R_{2}\right)$.

3) By Corollary 4.8, $\Gamma^{\prime}\left(\mathbb{Z}_{2} \times \mathbb{F}\right)$ is a star graph and so $\mathrm{g}\left(\Gamma^{\prime}\left(R_{1} \times R_{2}\right)\right)=\infty$.

4) First, assume that $\left|U\left(R_{2}\right)\right|>1$. Let $a \in W^{*}\left(R_{2}\right)$ and $1 \neq b \in U\left(R_{2}\right)$. Now, by Remarks 4.6 and 4.9 , we have the cycle $(0,1)-(1,0)-(0, b)-(1, a)-(0,1)$ in $\Gamma^{\prime}\left(R_{1} \times R_{2}\right)$. In the case that $\mid U\left(R_{2}\right)=1$, if $\Gamma^{\prime}\left(R_{2}\right)$ is not totally disconnected, then by part 1$)$,

$g\left(\Gamma^{\prime}\left(R_{1} \times R_{2}\right)\right)=3$. So, assume that there is no adjacency in $\Gamma^{\prime}\left(R_{2}\right)$. In this situation, we first show that $\Gamma^{\prime}\left(R_{1} \times R_{2}\right)$ is a bipartite graph. To this end, set

$V_{1}:=\left\{(0, a) \mid a \in R_{2}^{*}\right\}$ and $V_{2}:=\left\{(1, b) \mid b \in W\left(R_{2}\right)\right\}$. Clearly, $W^{*}\left(R_{1} \times R_{2}\right)=V_{1} \cup V_{2}$. Also, by Lemma 4.3, no two vertices in $V_{1}$ (or $V_{2}$ ) are adjacent. So $\Gamma^{\prime}\left(R_{1} \times R_{2}\right)$ is a bipartite graph, and thus $g\left(\Gamma^{\prime}\left(R_{1} \times R_{2}\right)\right)=4$ or $\infty$. Now, by Remark 4.9, $(0,1)$ is adjacent to all vertices $(1, b)$ in $\Gamma^{\prime}\left(R_{1} \times R_{2}\right)$, where $b \in W\left(R_{2}\right)$, and also $(1,0)$ is adjacent to all vertices $(0, a)$ in $\Gamma^{\prime}\left(R_{1} \times R_{2}\right)$, where $a \in R_{2}^{*}$. Hence, if there exist an element $a \in R_{2}^{*} \backslash\{1\}$ and $b \in W^{*}\left(R_{2}\right)$ such that $(0, a)$ is adjacent to $(1, b)$ in $\Gamma^{\prime}\left(R_{1} \times R_{2}\right)$, then $g\left(\Gamma^{\prime}\left(R_{1} \times R_{2}\right)\right)=4$. Otherwise, the girth of the cozero-divisor graph $\Gamma^{\prime}\left(R_{1} \times R_{2}\right)$ is infinity.

The following example presents a ring $R_{1} \times R_{2}$ with $\left|U\left(R_{2}\right)\right|=1$ which satisfies parts 1) and 4) of Theorem 4.13. This shows that all cases in the proof of the last part of Theorem 4.13, can occur.

Example 4.14 Let $R_{1}=\mathbb{Z}_{2}$ and $R_{2}=\mathbb{Z}_{2} \times \mathbb{Z}_{2}$. Then $\left|U\left(\mathbb{Z}_{2} \times \mathbb{Z}_{2}\right)\right|=1$ and by Proposition $4.11, \Gamma^{\prime}\left(\mathbb{Z}_{2} \times \mathbb{Z}_{2}\right)$ is complete. Hence, by Theorem 4.13 (i), we have that $g\left(\Gamma^{\prime}\left(\mathbb{Z}_{2} \times\left(\mathbb{Z}_{2} \times \mathbb{Z}_{2}\right)\right)\right)=3$.

\section{Acknowledgements}

The authors are deeply grateful to the referee for careful reading of the manuscript and helpful suggestions.

\section{REFERENCES}

[1] I. Beck, "Coloring of Commutative Rings," Journal of Algebra, Vol. 116, No. 1, 1988, pp. 208-226. doi:10.1016/0021-8693(88)90202-5

[2] D. F. Anderson and P. S. Livingston, "The Zero-Divisor Graph of a Commutative Ring,” Journal of Algebra, Vol. 217, No. 2, 1999, pp. 434-447. doi:10.1006/jabr.1998.7840

[3] M. Axtell, J. Coykendall and J. Stickles, "Zero-Divisor Graphs of Polynomials and Power Series over Commutative Rings,” Communications in Algebra, Vol. 33, No. 6, 2005, pp. 2043-2050. doi:10.1081/AGB-200063357

[4] T. G. Lucas, “The Diameter of a Zero Divisor Graph,” Journal of Algebra, Vol. 301, No. 1, 2006, pp. 174-193. doi:10.1016/j.jalgebra.2006.01.019 
[5] M. Axtell, J. Stickles and J. Warfel, "Zero-Divisor Graphs of Direct Products of Commutative Rings," Houston Journal of Mathematics, Vol. 32, No. 4, 2006, pp. 985-994.

[6] M. Afkhami and K. Khashyarmanesh, "The Cozero-Divisor Graph of a Commutative Ring," Southeast Asian Bulletin of Mathematics, Vol. 35, No. 5, 2011, pp. 753762.
[7] G. Chartrand and O. R. Oellermann, "Applied and Algorithmic Graph Theory,” McGraw-Hill, Inc., New York, 1993.

[8] J. A. Bondy and U. S. R. Murty, "Graph Theory with Applications,” American Elsevier, New York, 1976. 